\title{
Best linear forecast of volatility in financial time series
}

\author{
M. I. Krivoruchenko \\ Institute for Theoretical and Experimental Physics, B. Cheremushkinskaya 25 \\ 117259 Moscow, Russia \\ Institut für Theoretische Physik, Universität Tübingen, Auf der Morgenstelle 14 \\ D-72076 Tübingen, Germany and \\ Metronome-Ricerca sui Mercati Finanziari, C. so Vittorio Emanuele 84 \\ 10121 Torino, Italy
}

\begin{abstract}
The autocorrelation function of volatility in financial time series is fitted well by a superposition of several exponents. Such a case admits an explicit analytical solution of the problem of constructing the best linear forecast of a stationary stochastic process. We describe and apply the proposed analytical method for forecasting volatility. The leverage effect and volatility clustering are taken into account. Parameters of the predictor function are determined numerically for the Dow Jones 30 Industrial Average. Connection of the proposed method to the popular ARCH models is discussed.

PACS numbers: 89.65.Gh, 89.75.Da, 02.50.Ng, 02.50.Sk
\end{abstract}

\section{INTRODUCTION}

The methods developed in studying complex physical systems have been successfully applied throughout decades to analyze financial data 1, 2, 3]. The quantitative study of financial data continue to attract the growing interest motivated by the existence of universal features in the dynamics of different markets, such as power-law tails of the return distributions [4, 5, 6, 7, 8, 9, 10, 11], scaling as a first approximation [3] and deviations from scaling of the empirical return distributions [5, 8, 12], volatility clustering [12, 13], and leverage effect [14, 15, 16. The phenomenological and microscopic models $68,9,10,11,19,20,21,22$ have been proposed to explain the established stylized facts. The field of research connected to modeling financial markets has been named Econophysics.

A stock's volatility represents the simplest measure of its riskiness or uncertainty. Formally, the volatility is the annualized standard deviation of the stock's returns during the period of interest. The random walk model proposed by Bachelier in 1900 year [1] presupposes a constant volatility. There is an ample empirical evidence, however, that the volatility is not a constant, but represents a random variable. Two well established stylized facts concerning the volatility are long ranged volatility-volatility correlations that are also known as volatility clustering [13] and return-volatility correlations that are also known as leverage effect [14, 15].

The volatility is a key variable to control risk measures associated with the dynamics of prices of financial assets. The implied volatility extracted from options prices represents a market estimate of future volatility. A pure exposure to future volatility is provided by the volatility swaps [17, 18]. The volatility enters all options pricing models, so its knowledge has a great value for estimate of the equilibrium options state-price distributions.

The volatility clustering manifests itself in the occurrence of large changes of the index at neighboring times (observed localized outbursts). The leverage effect has its origin in the observed negative correlation between the past returns and future volatility. The possible explanation to this effect [14, 15, 16] is due to the fact that negative returns increase financial leverage and extend the risk for investors and thereby a stock's volatility. A statistical study 23. demonstrates clearly that the leverage effect is one-directional: past returns correlate with future volatility only.

In this paper, we propose an analytical method to evaluate future volatility as a linear function of the lagged volatility and lagged returns. The method takes the volatility clustering and leverage effect into account and provides for stationary stochastic processes the smallest forecasting error in the class of all linear functions. In this precise sense, we talk on the best linear forecast (BLF) of the volatility.

The BLF problem for a stationary stochastic process was formulated and solved by Kolmogorov 24 in 1941 year and Wiener [25] in 1949 year. A modern review of the BLF methods can be found in Ref. [26]. We apply these methods to construct the BLF volatility function for the Dow Jones 30 Industrial Average (DJIA).

The outline of the paper is as follows: In the next Sect., we remove the leverage effect from the original time series to work with a reduced volatility $\chi(t)$ that has by definition a vanishing covariance with the past returns. The spectral density of a stochastic process can be factorized, $f(\omega)=|\varphi(\omega)|^{2}$, if its correlation function represents a superposition of the exponential functions. An explicit expression is derived for the amplitude $\varphi(\omega)$. The analytical properties of the amplitude $\varphi(\omega)$ in the complex $\omega$-plane are important to provide an explicit representation of the predictor function. In Sect. 3, the BLF problem is analyzed further to account for the reduced volatility clustering and to construct the BLF function. In Sect. 4, we fit 100+ years of data of the daily historical volatility of the DJIA in order to determine parameters of the BLF function. Numerical estimates are given to illustrate the developed method. The minimization 
of the forecasting error for the reduced volatility predictor function is shown to be equivalent to the minimization of the forecasting error of the original volatility time series. An explicit expression for the forecasting error is given. In Conclusion, a connection of the BLF method with the ARCH models [27, 28, 29, 30], in which future variance is also represented as a linear combination of the past observables, is discussed.

\section{FACTORIZATION OF SPECTRAL DENSITY}

The evolution of a market index value or a stock price $S(t)$ is described by equation (see e.g. [31]):

$$
\frac{d S(t)}{S(t)}=\mu d t+d \psi(t)
$$

The value $d \psi(t)$ is a noise added to the path followed by $S(t)$, with the expectation value $\mathrm{E}[d \psi(t)]=0$ and the variance of $\operatorname{Var}[d \psi(t)]=\sigma(t)^{2} d t$. The volatility $\sigma(t)$ represents a generic measure of the magnitude of market fluctuations. We consider a discrete version of the random walk problem by setting $d t=1, d \psi(t)=\xi(t)$, and $d S(t)=S(t)-S(t-1)$. The sampling intervals are enumerated by integer time parameter $t$.

The volatility $\sigma(t)$ is a hidden variable and its extraction form the market observables is a separate difficult task. The possible estimator $\eta(t)=|\xi(t)|$ of the volatility is defined in terms of returns

$$
\xi(t)=(S(t)-S(t-1)) S(t)^{-1}-\mu .
$$

In what follows, the term "volatility" refers to the estimator $\eta(t)=|\xi(t)|$, the annualizing factor will not apply. A use of the variance estimator $v(t)=|\xi(t)|^{2}$ would complexify the problem due to divergences connected to the existence of power-law tails ("variance of variance" is infinite, $\operatorname{Var}\left[\xi^{2}\right]=\infty$, since $d F(\xi) \sim d \xi / \xi^{4}$ at $\xi \gg 1$, see e.g. [7]). At large time scales, different estimators are expected to be close to volatility $\sigma(t)$ and to each other. The problem of efficiency of volatility estimators is postponed for other studies.

It is usually assumed that financial time series constitute stationary stochastic processes the autocorrelation functions of which depend on the relative time only. The stock evolution problem is assumed therefore to be invariant with respect to time translations.

First, we remove from the time series $\eta(t)$ the leverage effect using the variable $\chi(t)$ :

$$
\chi(t)=\eta(t)-\sum_{s} \operatorname{Cov}[\eta(0), \xi(-s)] \operatorname{Var}^{-1}[\xi] \xi(t-s) .
$$

The decomposition (3) has a predictive power, since $\operatorname{Cov}[\eta(t), \xi(t-s)] \sim \theta(s)$, so $\chi(t)$ depends on the lagged price increments only. Note that $\mathrm{E}[\eta]=\mathrm{E}[\chi]$, since $\mathrm{E}[\xi]=0$. Due to the definition (3) and in virtue of equation

$$
\operatorname{Cov}[\xi(t), \xi(s)]=\delta_{t s} \operatorname{Var}[\xi]
$$

that holds true for sampling intervals greater than 20 Min. 7, 12], we have

$$
\operatorname{Cov}[\chi(t), \xi(s)]=0 \text {. }
$$

The reduced volatility $\chi(t)$ does not experience the leverage effect. So, its predictor depends on the past $\chi(t)$ only. It is possible therefore to focus on the volatility clustering only, while the leverage effect is taken into account explicitly through Eq. (3).

The autocorrelation function $B(t)=\operatorname{Cov}[\chi(t), \chi(0)] / \mathrm{E}\left[\chi^{2}\right]$ of a stationary stochastic process $\chi(t)$ can be fitted in many cases by a superposition of exponents

$$
B(t)=\sum_{i=1}^{n} d_{i} e^{-\alpha_{i}|t|}
$$

The best linear forecast of the observable $\chi(t)$ in such a case simplifies substantially. The case $n=1$ is discussed in Ref. [26]. We provide a solution of the BLF problem for arbitrary values of $n$.

The spectral density $f(\omega)$ of the stochastic process $\chi(t)$ is given by the Fourier transform of the correlations coefficient (6):

$$
f(\omega)=\sum_{t=-\infty}^{+\infty} e^{-i \omega t} B(t)=\sum_{i=1}^{n} d_{i} \frac{1-e^{-2 \alpha_{i}}}{\left(1-e^{-\alpha_{i}} u\right)\left(1-e^{-\alpha_{i}} \frac{1}{u}\right)}
$$


where $u=\exp (-i \omega)$. The function $f(\omega)$ can be represented in the form

$$
f(\omega)=P_{n-1}(\phi)\left(\prod_{i=1}^{n}\left(1-e^{-\alpha_{i}} u\right)\left(1-e^{-\alpha_{i}} \frac{1}{u}\right)\right)^{-1} .
$$

where $\phi=\frac{1}{2}\left(u+\frac{1}{u}\right)$ and

$$
\begin{aligned}
P_{n-1}(\phi) & =2^{n} \exp \left(-\sum_{i=1}^{n} \alpha_{i}\right) \sum_{i=1}^{n} d_{i} \sinh \left(\alpha_{i}\right) \prod_{k \neq i}^{n}\left(\cosh \left(\alpha_{i}\right)-\phi\right) \\
& =D_{n} 2^{n-1} \exp \left(-\sum_{i=1}^{n-1} \nu_{i}\right) \prod_{i=1}^{n-1}\left(\cosh \left(\nu_{i}\right)-\phi\right)
\end{aligned}
$$

The amplitude $\varphi(u)$ such that $f(\omega)=\varphi(u) \varphi(u)^{*}$ can be chosen to be analytical, rational and regular at $|u|<1$ :

$$
\varphi(u)=D_{n}^{1 / 2} \prod_{i=1}^{n-1}\left(1-e^{-\nu_{i}} u\right)\left(\prod_{i=1}^{n}\left(1-e^{-\alpha_{i}} u\right)\right)^{-1}
$$

The additive representation

$$
\varphi(u)=\sum_{i=1}^{n} c_{i} \frac{1}{1-\beta_{i} u}
$$

is completely equivalent to the multiplicative representation (10). Here, $\beta_{i}=e^{-\alpha_{i}}$ and

$$
\begin{aligned}
D_{n} & =2 \exp \left(-\sum_{i=1}^{n} \alpha_{i}+\sum_{i=1}^{n-1} \nu_{i}\right) \sum_{i=1}^{n} d_{i} \sinh \left(\alpha_{i}\right), \\
c_{i} & =D_{n}^{1 / 2} \prod_{k=1}^{n-1}\left(e^{-\alpha_{i}}-e^{-\nu_{k}}\right)\left(\prod_{k \neq i}^{n}\left(e^{-\alpha_{i}}-e^{-\alpha_{k}}\right)\right)^{-1}, \\
\cosh \left(\nu_{i}\right) & =\phi_{0}^{i}
\end{aligned}
$$

where $\phi_{0}^{i}$ are $n-1$ roots of equation $P_{n-1}\left(\phi_{0}^{i}\right)=0$. For $n=1, c_{1}=d_{1}^{1 / 2} \sqrt{1-\beta_{1}^{2}}$. The analytical solutions for $\nu_{i}$ exist up to $n=5$.

The knowledge of the Fourier transform of the autocorrelation function is not sufficient for a complete reconstruction of the Fourier transform of the stochastic process $\chi(t)$. If the autocorrelation function represents a superposition of the exponents (6), the problem admits a solution $\varphi(u)$, such that $f(u)=|\varphi(u)|^{2}$, in the class of rational functions. If we require further that the function $\varphi(u)$ be regular at $|u| \leq 1$, an unambiguous solution $\varphi(u)$ can be provided. This solution coincides with the Fourier transform of the time series $\chi(t)$ up to a phase factor. It is remarkable, that we need not to know the phase, since all the relevant information is contained in the spectral density $f(\omega)$. The BLF problem simplifies then considerably due to the special analytical properties of the function $\varphi(u)$.

\section{BLF FUNCTION}

The correlation function corresponding to the spectral density (7) can be found from the inverse Fourier transform. In case of (10), we consider first $t>0$ :

$$
B(t)=\int_{-\pi}^{\pi} e^{i \omega t} \varphi(\omega) \varphi(-\omega) \frac{d \omega}{2 \pi}=-\int_{\mathcal{C}_{r}} \frac{1}{u^{t+1}} \varphi(u) \varphi\left(\frac{1}{u}\right) \frac{d u}{2 \pi i}
$$

where $\mathcal{C}_{r}=\left\{e^{-i \omega}, \omega=-\pi \ldots \pi\right\}$. The poles of $\varphi(u)$ are located at $|u| \geq R=\min \left\{e^{\alpha_{i}}\right\}$, while the poles of $\varphi\left(\frac{1}{u}\right)$ are located at $|u| \leq 1 / R$. We move the contour $\mathcal{C}_{r}$ to infinity and get

$$
B(t)=\sum_{i=1}^{n} \sum_{k=1}^{n} \beta_{i}^{|t|} \frac{c_{i} c_{k}}{1-\beta_{i} \beta_{k}}
$$


Comparison with Eq.(6) gives

$$
d_{i}=\sum_{k=1}^{n} \frac{c_{i} c_{k}}{1-\beta_{i} \beta_{k}}
$$

The same result (14) comes out at $t<0$. For $n=1, B(t)=\beta_{1}^{|t|} c_{1}^{2} /\left(1-\beta_{1}^{2}\right)$.

A stochastic process $\chi(t)$ can be represented as a linear combination of a normally distributed uncorrelated sequence $\zeta(t) \sim N\left(0, \sigma_{\chi}\right)$ with $\sigma_{\chi}^{2}=\mathrm{E}\left[\chi^{2}\right]$

$$
\chi(t)=\mathrm{E}[\chi]+\sum_{s=0}^{+\infty} C(s) \zeta(t-s)
$$

provided that the spectral function admits the factorization and the amplitude $\varphi(u$ ) is regular at $|u|=1<R$ (see e.g. [26]). The expansion coefficients equal

$$
C(t)=\sum_{i=1}^{n} c_{i} \beta_{i}^{t}
$$

It is remarkable that only retarded $\zeta(s)$ enter the summation in Eq. (16). This is a consequence of the convergence of the Taylor expansion of the amplitude $\varphi(u)$ at $|u|=1$, which is in turn a consequence of the analyticity at $|u|<R$ : The convergence radius of the expansion is associated with the first pole at $\left|u_{1}\right|=R$. The stationary stochastic process $\chi(t)$ can be interpreted as a result of filtering the normal sequence $\zeta(t)$.

The BLF function for the time horizon $\tau$ has the form [26]

$$
\hat{\chi}_{\tau}(t)=\mathrm{E}[\chi]+\sum_{s=\tau}^{+\infty} \frac{\Xi_{\tau}(0)^{(s)}}{s !}(\chi(t-s)-\mathrm{E}[\chi]) .
$$

The weight coefficients $\Xi_{\tau}(0)^{(s)}$ are derivatives of the function $\Xi_{\tau}(u)=\varphi_{\tau}(u) / \varphi(u)$ at $u=0$. Here,

$$
\varphi_{\tau}(u)=\sum_{s=\tau}^{+\infty} C(t) u^{s}=\sum_{i=1}^{n} c_{i}\left(\beta_{i} u\right)^{\tau} \frac{1}{1-\beta_{i} u} .
$$

For constructing a linear prognosis function, the overall normalization factor in $B(t)$ is not important, since it drops out from the ratio $\varphi_{\tau}(u) / \varphi(u)$. In virtue of Eq. (5),

$$
\operatorname{Cov}\left[\hat{\chi}_{\tau}(t), \xi(s)\right]=0 .
$$

In terms of the stochastic process $\zeta(t)$, the BLF function looks like

$$
\hat{\chi}_{\tau}(t)=\mathrm{E}[\chi]+\sum_{s=\tau}^{+\infty} C(s) \zeta(t-s)
$$

At $s=\tau$, we obtain

$$
\frac{\Xi_{\tau}(0)^{(\tau)}}{\tau !}=\frac{\sum_{i=1}^{n} c_{i} e^{-\alpha_{i} \tau}}{\sum_{i=1}^{n} c_{i}}
$$

and at $l=s-\tau>0$

$$
\frac{\Xi_{\tau}(0)^{(s)}}{s !}=D_{n}^{-1 / 2} \sum_{j=1}^{n-1} e^{-\nu_{j}(l-1)}\left(\prod_{i \neq j}^{n-1}\left(e^{-\nu_{j}}-e^{-\nu_{i}}\right)\right)^{-1} \sum_{i=1}^{n} c_{i} e^{-\alpha_{i} \tau} \prod_{k \neq i}^{n}\left(e^{-\nu_{j}}-e^{-\alpha_{k}}\right) .
$$

The last two equations complete solution of the BLF problem for the case when the correlation function is a superposition of the exponent functions (6). The $n(n-1)$ terms in the right side of Eq.(22) are not all positive definite. 


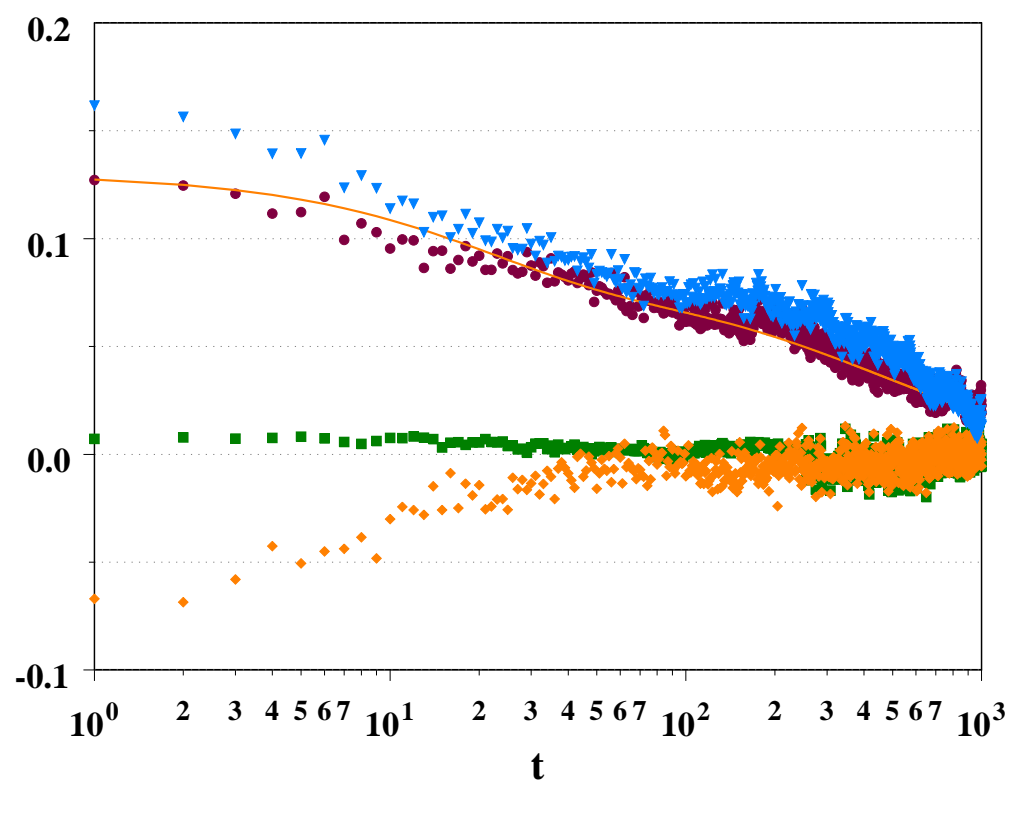

FIG. 1: Empirical correlation coefficients $\operatorname{Corr}[\eta(t), \eta(0)]$, Corr $[\eta(t), \xi(0)]$ and $\operatorname{Corr}[\chi(t), \chi(0)]$, Corr $[\chi(t), \xi(0)]$ versus the number of trading days $t$ of the Dow Jones 30 Industrial Average. The reduced volatility $\chi(t)$ is defined through Eq. (3). The correlation coefficients are calculated using 100+ years of the daily quotes, starting on the May 26, 1896 and ending on the December 31, 1999 (i.e. a total of 28507 trading days). The values of Corr $[\eta(t), \eta(0)]$ and Corr $[\eta(t), \xi(0)]$ are denoted, respectively, by triangles and diamonds. The values of $\operatorname{Corr}[\chi(t), \chi(0)]$ and $\operatorname{Corr}[\chi(t), \xi(0)]$ are denoted by circles and boxes. The solid curve is the exponential fit (6) with parameters given in Table 1. It is seen that $\operatorname{Corr}[\chi(t), \xi(0)] \approx 0$. The leverage effect is thus removed from $\chi(t)$.

TABLE I: Parameters $d_{i}$ and $\alpha_{i}$ entering the fit of the autocorrelation function (6) of the reduced volatility, parameters $\nu_{i}$ which determine the roots of equation $P_{n-1}\left(\phi_{0}\right)=0$, and parameters $c_{i}$ which determine the additive representation (11) of the function $\varphi(u)$. The value of $D_{4}$ represents the normalization constant according to Eq.(12).

\begin{tabular}{llllllll}
\hline \hline$d_{1}$ & 0.40 & $\alpha_{1}$ & $+\infty$ & $\nu_{1}$ & .002257 & $c_{1}$ & .606241 \\
$d_{2}$ & 0.05 & $\alpha_{2}$ & $1 / 20$ & $\nu_{2}$ & .012107 & $c_{2}$ & .038233 \\
$d_{3}$ & 0.03 & $\alpha_{3}$ & $1 / 250$ & $\nu_{3}$ & .125302 & $c_{3}$ & .007857 \\
$d_{4}$ & 0.05 & $\alpha_{4}$ & $1 / 1000$ & $D_{4}$ & .435341 & $c_{4}$ & .007473 \\
\hline \hline
\end{tabular}

\section{PARAMETERS OF BLF VOLATILITY FUNCTION FOR DOW JONES 30 INDUSTRIAL AVERAGE}

Let us apply the BLF method to forecasting the volatility for the DJIA. The daily returns are defined by Eq.(2) where $S(t)$ are the DJIA index close values, the volatility equals $\eta(t)=|\xi(t)|$, and $\chi(t)$ is defined by Eq.(3). In Fig.1, we show the empirical values of the correlation coefficients $\operatorname{Corr}[\eta(t), \eta(0)]$, $\operatorname{Corr}[\eta(t), \xi(0)]$ and $\operatorname{Corr}[\chi(t), \chi(0)]$, Corr $[\chi(t), \xi(0)]$ and the exponential fit of the correlation coefficient Corr $[\chi(t), \chi(0)]$ versus the time lag $t$. Let us remind that $\operatorname{Corr}[A, B]=\operatorname{Var}[A, B] / \sqrt{\mathrm{E}\left[A^{2}\right] \mathrm{E}\left[B^{2}\right]}$ and $-1 \leq \operatorname{Corr}[A, B] \leq 1$. To calculate $\chi(t)$, we run the summation over $s$ in Eq.(3) from 0 to 250 and use the empirical correlation function of $\eta(t)$ and $\xi(s)$ without additional smearing. Up to 
TABLE II: The weight coefficients (21) and 22) of the BLF function for some values of the parameters $l$ and $\tau$. Here, $\tau$ is the forecast horizon, $l=s-\tau$ is the number of trading day entering the predictor function starting from the most recent day.

\begin{tabular}{lcccc}
\hline \hline$l$ & $\tau=1$ & $\tau=2$ & $\tau=10$ & $\tau=100$ \\
\hline 0 & .07830 & .07555 & .05780 & .01862 \\
1 & .06942 & .06702 & .05150 & .01710 \\
2 & .06158 & .05949 & .04594 & .01575 \\
10 & .02432 & .02366 & .01941 & .00916 \\
\hline \hline
\end{tabular}

$t=250$, the correlation coefficient $\operatorname{Corr}[\chi(t), \xi(0)]$ is less noisy as compared with other correlators. The parameters $d_{i}$ and $\alpha_{i}$ are listed in Table 1. The equation $P_{3}(\phi)=0$ determines the parameters $\nu_{i}(i=1,2,3)$ according to Eq. (9). Using Eqs.(12), we find the values of $D_{4}$ and $c_{i}$, which we also show in Table 1. One can check that Eq. (15) is satisfied. The correlation coefficient $\operatorname{Corr}[\chi(t), \chi(0)]$ drops from $0.53\left(=\sum_{i=1}^{4} d_{i}\right)$ to $0.13\left(=\sum_{i=2}^{4} d_{i}\right)$ when $t$ changes by one unit from $t=0$ to $t=1$. The value of $\alpha_{1}$ is therefore large and can be fixed by considering high-frequency data only. The results shown on Fig. 1 and in Table 1 and Table 2 are obtained for $\alpha_{1}=+\infty$. The values of $1 / \alpha_{i}$ for $i=2,3,4$ equal to about one month, one year, and four calendar years, respectively.

The weight coefficients $\Xi_{\tau}(0)^{(s)}$ can be found with the use of Eq.(21) at $s=\tau$ and Eq.(22) at $s>\tau$. We show values of the weight coefficients divided by $s$ ! in Table 2 for $l=s-\tau=0,1,2$, and 10 and $\tau=1,2,10$, and 100 .

The BLF volatility function looks like

$$
\hat{\eta}_{\tau}(t)=\mathrm{E}[\chi]+\sum_{s=\tau}^{+\infty} \frac{\Xi_{\tau}(0)^{(s)}}{s !}(\chi(t-s)-\mathrm{E}[\chi])+\sum_{s=\tau}^{+\infty} \operatorname{Cov}[\eta(0), \xi(-s)] \operatorname{Var}^{-1}[\xi] \xi(t-s)
$$

where the unknown future returns set qual to zero: $\xi(t-s) \rightarrow \mathrm{E}[\xi(t-s)]=0$ for $0 \leq s<\tau$.

Using Eqs.(4), (5), and (19), one gets

$$
\mathrm{E}\left[\left(\hat{\eta}_{\tau}(t)-\eta(t)\right)^{2}\right]=\mathrm{E}\left[\left(\hat{\chi}_{\tau}(t)-\chi(t)\right)^{2}\right]+\sum_{s=0}^{\tau-1} \operatorname{Cov}[\eta(0), \xi(-s)]^{2} \operatorname{Var}^{-1}[\xi],
$$

so the minimization of the $\hat{\chi}_{\tau}(t)$ error according to Eq. (18) is equivalent to the minimization of the $\hat{\eta}_{\tau}(t)$ error. Using decompositions (16) and (20), the $\hat{\chi}_{\tau}(t)$ error can be evaluated as

$$
\mathrm{E}\left[\left(\hat{\chi}_{\tau}(t)-\chi(t)\right)^{2}\right]=\mathrm{E}\left[\chi^{2}\right] \sum_{s=0}^{\tau-1} C^{2}(s)=\mathrm{E}\left[\chi^{2}\right] \sum_{i=1}^{n} \sum_{k=1}^{n} c_{i} c_{k} \frac{1-e^{-\left(\alpha_{i}+\alpha_{k}\right) \tau}}{1-e^{-\left(\alpha_{i}+\alpha_{k}\right)}} .
$$

(There is a misprint in Eq.(10.2) of Ref. [26]) At $\tau \rightarrow+\infty, \mathrm{E}\left[\left(\hat{\chi}_{\tau}(t)-\chi(t)\right)^{2}\right] \rightarrow \mathrm{E}\left[\chi^{2}\right] \sum_{i=1}^{n} d_{i}=\operatorname{Var}[\chi]$, in agreement with the fact that $\hat{\chi}_{\tau}(t) \rightarrow \mathrm{E}[\chi]$. The arguments of such a kind do not apply to the variance estimator $v(t)=|\xi(t)|^{2}$, since $\operatorname{Var}[v]=\infty$ due to the power-law tails of the return distributions [].

Nonlinear models for volatility forecasting [19, 20], which take into account besides the volatility clustering and leverage effect also heavy tails of the returns distributions and the approximate scaling, represent an alternative class of the stochastic volatility models. The efficiency of such models can be tested in general using Monte Carlo simulations and/or backtests over historical data. The approach of Refs. [19, 20] is more general, since it allows a calculation of the probability density function of the volatility. The BLF method predicts the average volatility only. It can, however, be extended to forecasting $|\xi|^{a}$ for arbitrary $0<a$ such that $\mathrm{E}\left[|\xi|^{2 a}\right]<\infty$. If all moments $\mathrm{E}\left[|\xi|^{a}\right]$ of the future distribution are known, the reconstruction of the probability density function of the volatility must be possible within the BLF method also.

\section{CONCLUSION}

The BLF problem for a stationary stochastic process was formulated in 1941 year by Kolmogorov 24] and later by Wiener [25]. A modern review of the BLF methods can be found in Ref. [26]. In this paper, we reported an explicit analytical solution of the BLF problem for practically important case when the autocorrelation function represents 
a superposition of exponential functions. The autocorrelation function of the volatility in a financial time series is known to be fitted well by such a superposition. We applied the obtained results to construct the BLF volatility function for the DJIA.

The popular autoregressive conditional heteroskedasticity (ARCH) models of time dependent volatility, proposed by Engle [27] (for a review see [28, 29, 30]), describe the variance $\sigma(t)^{2}$ as a linear function of the past observables. The ARCH models are conceptually very close to the BLF approach. Eq.(23) expresses the forecasting volatility also as a linear function of the past volatility and past returns. Eq. (23) gives, however, the best linear forecast with the proved smallest forecasting error (25). The weight coefficients $\Xi_{\tau}(0)^{(s)}$ allow to evaluate the magnitude and number of terms needed for the ARCH models to quantify future variance with sufficiently good precision. The ARCH models receive an additional support and more general framework through the BLF formula (23).

The accurate estimates of the future volatility are important for risk management and options pricing. The BLF formula (23) represents an interest as the proved most accurate estimate in the class of all linear functions of the past volatility and past returns.

\section{Acknowledgments}

The author wishes to thank E. Alessio and V. Frappietro for several useful discussions and the Dow Jones Global Indexes for providing the DJIA historical quotes. This work has been supported in part by Federal Program of the Russian Ministry of Industry, Science and Technology No. 40.052.1.1.1112.

[1] L. Bachelier, Ann. Sci. Ercole Norm. Suppl. 3, 21 (1900).

[2] P. Levy, Théorie de l'Addition des Variables Aléatoires (Gauthier-Villars, Paris, 1937).

[3] B. B. Mandelbrot, J. Business 36, 294 (1963).

[4] P. C. Clark, Econometrica 41, 135 (1973).

[5] R. N. Mantegna and H. E. Stanley, Nature 376, 46 (1995).

[6] H. Geman, Risk 9, 145 (1996).

[7] P. Gopikrishnan, V. Plerou, L. A. Nunes Amaral, M. Meyer, and H. E. Stanley, Phys. Rev. E60, 5305 (1999).

[8] R. N. Mantegna and H. E. Stanley, An introduction to Econophysics: correlations and complexity in finance (Cambridge University Press, 2000).

[9] V. Plerou et al., Phys. Rev. E62, R3023-6 (2000).

[10] V. Plerou et al., Quantitative Finance 1, 262 (2001).

[11] E. Derman, Quantitative Finance 2, 282 (2002).

[12] R. Cont, Scaling and correlations in financial data, 1997. http://arXiv.org/abs/cond-mat/9705075

[13] Z. Ding, C. W. J. Granger and R. F. Engle, J. Empirical Finance 1, 83 (1993).

[14] F. Black, Proceedings of the 1976 Americal Statistical Association, Business and Economical Statistics Section, p. 177 (1976).

[15] J. C. Cox and S. A. Ross, J. Fin. Eco. 3, 145 (1976).

[16] A. A Christie, J. Fin. Eco. 10, 407 (1982).

[17] K. Demeterfi, E. Derman, M. Kamal and J. Zou, More than you ever wanted to know about volatility swaps, Goldman Sachs, Quantitative Strategies Research Notes, 1999. http://www.ederman.com/emanuelderman/GSQSpapers/volswaps.pdf

[18] P. Carr and D. Madan, Towards a theory of volatility swaps, 2002. http://petercarr.net]

[19] E. Alessio, V. Frappietro, M. I. Krivoruchenko, and L. J. Streckert, Multivariate distribution of returns in financial time series, 2003. In: Proceedings of the International Conference of Computational Methods in Sciences and Engineering 2003 (ICCMSE 2003), Ed. T.E. Simos (World Scientific Publishing Co., Singapore, 2003), pp. 323-326 http://arXiv.org/abs/cond-mat/0310300

[20] M. I. Krivoruchenko, E. Alessio, V. Frappietro, and L. J. Streckert, Modeling stylized facts for financial time series, 2003. Talk given at the Conference "Applications of Physics in Financial Analysis 4", Warsaw, 13-15 November, 2003. http://arXiv.org/abs/cond-mat/0401009

[21] I. Giardina, J-P. Bouchaud, M. Mezard, Microscopic models for long ranged volatility correlations, 2001. http://arXiv.org/abs/cond-mat/0105076

[22] A. Krawiecki, J. A. Holyst and D. Helbing, Phys. Rev. Lett. 89, 158701 (2002).

[23] J-P. Bouchard, A. Matacz and M. Potters, Phys. Rev. Lett. 87, 228701 (2001).

[24] A. N. Kolmogorov, Stationary sequences in Hilbert space (in Russian), Byulleten' Moskovskogo Universiteta, 2, 1 (1941).

[25] N. Wiener, Extrapolation, interpolation and smoothing of stationary time series (John Wiley, New York, 1949).

[26] V. S. Korolyuk, N. I. Portenko, A. V. Skorokhod, A. F. Turbin, Handbook on probability theory and mathematical statistics (Nauka, Moscow, 1985).

[27] R. F. Engle, Econometrica 50, 987 (1982). 
[28] T. Bollerslev, U. Chou and K. Kroner, Journal of Econometrics 52, 5 (1992).

[29] F. Diebold and J. Lopez, Modeling Volatility Dynamics, Federal Reserve Bank of New York Research Paper 9522 (1995).

[30] J. Perello, J. Masoliver and N. Anento, A comparison between several correlated stochastic volatility models, 2003. http://arXiv.org/abs/cond-mat/0312121

[31] J. C. Hull, Options, futures, and other derivatives, 4-th edn. (Prentice Hall, New Jersey, 2000). 